Research Paper

\title{
Predictive Value of Echocardiographic Abnormalities and the Impact of Diastolic Dysfunction on In-hospital Major Cardiovascular Complications after Living Donor Kidney Transplantation
}

\author{
Eun Jung Kim, ${ }^{1,2}$ Suyon Chang, ${ }^{3}$ So Yeon Kim, ${ }^{1,2}$ Kyu Ha Huh, ${ }^{4}$ Soojeong Kang, ${ }^{1}$ Yong Seon Choi ${ }^{1,2}$ \\ 1. Department of Anesthesiology and Pain Medicine, Severance Hospital, Yonsei University College of Medicine, Seoul, Korea; \\ 2. Anesthesia and Pain Research Institute, Yonsei University College of Medicine, Seoul, Korea: \\ 3. Department of Radiology, Severance Hospital, Yonsei University College of Medicine, Seoul, Korea; \\ 4. Department of Transplantation Surgery, Severance Hospital, Yonsei University College of Medicine, Seoul, Korea.
}

$\square$ Corresponding author: Yong Seon Choi, MD, PhD. Department of Anesthesiology and Pain Medicine, Severance Hospital, Yonsei University College of Medicine, 50-1 Yonsei-ro, Seodaemun-gu, Seoul 120-752, Republic of Korea. Office Phone: +82-2-2228-2412 Fax: +82-2-2227-7897 E-mail: YSCHOI@yuhs.ac.

() Ivyspring International Publisher. Reproduction is permitted for personal, noncommercial use, provided that the article is in whole, unmodified, and properly cited. See http://ivyspring.com/terms for terms and conditions.

Received: 2016.04.05; Accepted: 2016.07.07; Published: 2016.07.18

\begin{abstract}
Patients with end-stage renal disease (ESRD) show characteristic abnormalities in cardiac structure and function. We evaluated the influence of these abnormalities on adverse cardiopulmonary outcomes after living donor kidney transplantation in patients with valid preoperative transthoracic echocardiographic evaluation. We then observed any development of major postoperative cardiovascular complications and pulmonary edema until hospital discharge. In-hospital major cardiovascular complications were defined as acute myocardial infarction, ventricular fibrillation/tachycardia, cardiogenic shock, newly-onset atrial fibrillation, clinical pulmonary edema requiring endotracheal intubation or dialysis. Among the 242 ESRD study patients, 9 patients (4\%) developed major cardiovascular complications, and 39 patients (16\%) developed pulmonary edema. Diabetes, ischemia-reperfusion time, left ventricular end-diastolic diameter (LVEDd), left ventricular mass index (LVMI), right ventricular systolic pressure (RVSP), left atrium volume index (LAVI), and high E/E' ratios were risk factors of major cardiovascular complications, while age, LVEDd, LVMI, LAVI, and high E/E' ratios were risk factors of pulmonary edema. The optimal E/E' cut-off value for predicting major cardiovascular complications was 13.0, showing $77.8 \%$ sensitivity and $78.5 \%$ specificity. Thus, the patient's E/E' ratio is useful for predicting in-hospital major cardiovascular complications after kidney transplantation. We recommend that goal-directed therapy employing E/E' ratio be enacted in kidney recipients with baseline diastolic dysfunction to avert postoperative morbidity. (http://Clinical Trials.gov number: NCT02322567)
\end{abstract}

Key words: living donor kidney transplantation, end-stage renal disease, diastolic dysfunction, pulmonary edema, tissue Doppler imaging.

\section{Introduction}

Advanced chronic kidney disease often results in adverse cardiovascular outcomes, often the leading causes of mortality in patients with end-stage renal disease (ESRD) [1]. ESRD patients on dialysis not only experience traditional cardiovascular risk factors, including hypertension, diabetes, and hyperlipidemia, but also hemodynamic overload and non-hemodynamic risk factors, such as biochemical and neurohormonal factors that promote chronic inflammation and fibrosis $[2,3]$.

Cardiac alterations in morphology and function, such as left ventricle (LV) hypertrophy, LV dilation, and systolic dysfunction, are predictors for uremic cardiomyopathy, which results in a 3-fold increased 
risk of heart failure [2,4]. With improved surgical techniques and immunosuppressive regimens, kidney transplantation is now considered the standard therapy to treat ESRD patients. Reports have shown that kidney transplantation normalizes cardiac alterations and leads to corresponding survival improvement in kidney transplant recipients with preoperative cardiac dysfunction. However, changes in diastolic dysfunction after transplantation are somewhat controversial in the literature [5-7], as they may persist or worsen even after transplantation $[6,8]$.

Among echocardiographic abnormalities, LV hypertrophy, which is frequently accompanied by cardiac fibrosis and subclinical diastolic dysfunction, develops early during chronic kidney disease progression [9-11]. In early ESRD, diastolic dysfunction with relatively preserved systolic function occurs in more than half of hemodialysis patients as revealed by tissue Doppler echocardiographic assessment $[12,13]$. Several studies have shown that diastolic dysfunction is associated with perioperative cardiopulmonary events in patients undergoing various types of surgery [11,14-16]. The ratio of early transmitral flow velocity to early diastolic velocity of the mitral annulus (E/E') is a reliable indicator of diastolic function that correlates well with LV filling pressure [17]. Even in ESRD patients on hemodialysis, the $\mathrm{E} / \mathrm{E}^{\prime}$ ratio can predict general and cardiac mortality because it is a relatively preload-independent parameter $[18,19]$. In recent years, preemptive or well-timed living donor kidney transplantation has been performed at higher levels of estimated glomerular filtration rate or in earlier stages of dialysis than it was previously, leading to a survival advantage [20]. It has not been thoroughly evaluated whether echocardiographic parameters, including reliable indicators of diastolic function, can predict cardiopulmonary complications after kidney transplantation in patients in early dialysis. Therefore, we aimed to analyze the implications of echocardiographic parameters and diastolic dysfunction on major postoperative cardiovascular complications and pulmonary edema in ESRD patients undergoing living donor kidney transplantation.

\section{Patients and Methods}

\section{Study participants}

This prospective and observational study was conducted between January 2012 and September 2015 at Yonsei university hospital. After approval from the Institutional Review Board, we registered the study with http://clinicaltrials.gov (NCT02322567). We enrolled patients with valid preoperative transthoracic echocardiographic evaluation within 2 months before surgery, aged 20-70 years, classified as American Society of Anesthesiologists Physical Status 3 or 4 , and scheduled to undergo living donor kidney transplantation. Patients with severe valvular dysfunction [21], history of myocardial infarction, more than minimal pericardial effusion, non-sinus rhythm, previous kidney transplantation, and multiple organ transplantation were excluded.

\section{Assessment of cardiac structure and function}

Before surgery, each patient underwent routine transthoracic echocardiography to obtain tissue Doppler measurements the day after the patients' regular hemodialysis schedule. We calculated their LV ejection fraction with the biplane Simpson method and measured their interventricular septal diameter, LV end-diastolic diameter (LVEDd), LV mass, and posterior wall diameter according to American Society of Echocardiography guidelines [22]. We measured LV diastolic function using the ratio of peak early and late (atrial) mitral inflow (E/A) and the $\mathrm{E} / \mathrm{E}^{\prime}$ ratio with echocardiography $[16,23]$. We estimated right ventricular systolic pressure (RVSP) from the tricuspid regurgitation velocity using the modified Bernoulli equation.

\section{Anesthetic management}

Anesthesia was induced with propofol 1.5-2 $\mathrm{mg} / \mathrm{kg}$, remifentanil $0.5-1 \mu \mathrm{g} / \mathrm{kg}$, and rocuronium bromide $0.6 \mathrm{mg} / \mathrm{kg}$. Subsequently, a radial artery catheter and an internal jugular central venous catheter were inserted. Anesthesia was maintained with desflurane 0.8-1.0 minimal alveolar concentration in $50 \% \mathrm{O}_{2} /$ air mixture and remifentanil 0.05-0.15 $\mathrm{\mu g} / \mathrm{kg} / \mathrm{min}$. Acetate-buffered balanced crystalloid solution and total $750 \mathrm{~mL}$ of $5 \%$ albumin were given throughout the surgery. Any hypotensive episodes (greater than 20\% decrease in mean blood pressure (MBP) from the preoperative baseline value) were treated with $6 \mathrm{mg}$ of IV ephedrine and/or norepinephrine infusion. Irradiated filtered packed red blood cells were transfused when the hematocrit level dropped more than 25\% from baseline throughout the study period. The operation was performed in a standardized manner in all patients. Intraoperative hemodynamic parameters, including the MBP, heart rate (HR), central venous pressure $(\mathrm{CVP})$, and stroke volume variation (SVV), were recorded at four different time points: $10 \mathrm{~min}$ after induction of anesthesia (baseline), 60 minutes after the start of surgery, 10 minutes after reperfusion of the kidney graft, and at the end of surgery. Arterial blood gas (ABG) analyses were performed at the same time points. We also noted the duration of surgery, kidney 
graft ischemia-reperfusion time, intraoperative fluid balance, and the number of patients receiving any inotropic or vasopressors. Demographic, clinical, echocardiographic, and laboratory data were obtained directly from each patient's electronic medical record. All transplant recipients received protocol-driven, standardized immunosuppressive strategies.

\section{Outcome Measures}

The occurrence of in-hospital major cardiovascular complications after kidney transplantation was the primary endpoint of our study, which included acute myocardial infarction, ventricular fibrillation/tachycardia, cardiogenic shock, and newly-onset atrial fibrillation, as well as clinical pulmonary edema requiring endotracheal intubation or dialysis $[9,24,25]$. The secondary endpoint was the development of postoperative pulmonary edema as indicated by radiological evidence during hospitalization, which was evaluated by a designated radiologist blinded to clinical and echocardiographic information from each patient. Serial electrocardiograms and chest radiographs were obtained before surgery, the first and/or second postoperative day, and whenever patients complained of any cardiopulmonary symptoms. We noted any event of delayed graft function (DGF), acute rejection episodes (ARE), and graft loss defined as follows: DGF resulted in dialysis within 1 week of transplantation, ARE included both biopsy-proven and clinically suspected acute rejection until the time of hospital discharge, and graft loss involved initiation of long-term dialysis therapy within 1 year after transplantation [26]. We evaluated postoperative kidney function based on serum levels of blood urea nitrogen and creatinine $(\mathrm{Cr})$, and estimated glomerular filtration rate (eGFR) based on the modification of diet in renal disease formula applied on postoperative days 1,2 , and 7 .

\section{Statistical analysis}

We performed statistical analyses using SPSS for Windows, version 20.0 (SPSS Inc, Chicago, IL). All data are expressed as means \pm standard deviation (SD), medians (interquartile range), or number of patients (percentage). We compared normally distributed continuous variables using an unpaired two-tailed Student's t-test and non-normal continuous variables using a Mann-Whitney $U$-test or Kruskal-Wallis test. We analyzed categorical data with a $\chi^{2}$ or Fisher's exact test where appropriate. We evaluated repeated measured variables, such as ABG values and postoperative renal function, using linear mixed models with Bonferroni correction. We performed univariate logistic regression analysis to calculate odds ratios for independent parameters associated with in-hospital major cardiovascular complications and postoperative pulmonary edema, and significant variables with $P$-value $<0.05$ were included in the subsequent multivariate logistic regression model. We then calculated the receiver-operating characteristic (ROC) curve to determine the most appropriate $\mathrm{E} / \mathrm{E}^{\prime}$ ratio cut-off value for occurrence of in-hospital major cardiovascular complications and evaluated its accuracy based on the area under the curve (AUC) using MedCalc version 9.3.6.0 (MedCalc Software, Belgium). A $P$-value less than 0.05 indicated statistical significance.

\section{Results}

Of the 597 adult patients who underwent living donor kidney transplantation during our study period, we identified 242 patients who fulfilled the inclusion and exclusion criteria. The participants' demographic and baseline clinical data, including preoperative transthoracic echocardiographic findings, are summarized in Table 1.

Table 1. Baseline characteristics and Echocardiographic data.

\begin{tabular}{|c|c|}
\hline Age (yr) & $44.7 \pm 11.6$ \\
\hline Male & $150(62)$ \\
\hline BMI $\left(\mathrm{kg} / \mathrm{m}^{2}\right)$ & $22.2 \pm 3.6$ \\
\hline \multicolumn{2}{|l|}{ Medical History } \\
\hline HTN & $197(81)$ \\
\hline DM & $51(21)$ \\
\hline CAOD & $8(3)$ \\
\hline COPD & $3(1)$ \\
\hline $\mathrm{HD} / \mathrm{PD}$ & $199(82) / 26(11)$ \\
\hline Duration of CRF (yr) & $1.5(0.5-5.3)$ \\
\hline Duration of RRT (months) & $2(1-14)$ \\
\hline Preoperative $\mathrm{Hb}(\mathrm{mg} / \mathrm{dL})$ & $10.2 \pm 1.5$ \\
\hline \multicolumn{2}{|l|}{ Operative Data } \\
\hline Op time (min) & $268.5 \pm 62.9$ \\
\hline I-R time (min) & $71.9 \pm 21.4$ \\
\hline \multicolumn{2}{|l|}{ Echocardiographic data } \\
\hline $\operatorname{LVEF}(\%)$ & $65.0 \pm 6.3$ \\
\hline LVESd (mm) & $33.9 \pm 4.4$ \\
\hline LVEDd (mm) & $50.9 \pm 4.7$ \\
\hline $\operatorname{LVMI}\left(\mathrm{g} / \mathrm{m}^{2}\right)$ & $117.5 \pm 32.1$ \\
\hline LV hypertrophy & $78(32)$ \\
\hline $\mathrm{E} / \mathrm{A}$ ratio $\geq 2$ & $7(3)$ \\
\hline $\mathrm{E} / \mathrm{E}^{\prime}$ ratio & $11.0 \pm 4.4$ \\
\hline$>15$ & $33(14)$ \\
\hline $8-15$ & $157(65)$ \\
\hline$<8$ & $52(21)$ \\
\hline RVSP (mmHg) & $26.2 \pm 8.2$ \\
\hline$\geq 35 \mathrm{mmHg}$ & $22(9)$ \\
\hline LAVI $\left(\mathrm{mL} / \mathrm{m}^{2}\right)$ & $31.4 \pm 11.5$ \\
\hline \multicolumn{2}{|c|}{$\begin{array}{l}\text { Numbers are expressed as means } \pm \mathrm{SD} \text {, medians (interquartile range), or numbers of } \\
\text { patients (percentage). }\end{array}$} \\
\hline \multicolumn{2}{|c|}{$\begin{array}{l}\text { BMI, body mass index; HD, hemodialysis; PD, peritoneal dialysis; } C R F \text {, chronic renal } \\
\text { failure; RRT, renal replacement therapy; Hb, hemoglobin; I-R time, ischemia-reperfusion } \\
\text { time, LVEF, LV ejection fraction; LVESd, LV end-systolic dimension; LVEDd, LV } \\
\text { end-diastolic dimension; LVMI, LV mass index; } \mathrm{E} \text { A, ratio of early (E) to late (A) } \\
\text { ventricular filling velocities; E/E' ratio, ratio of mitral peak velocity of early filling (E) to } \\
\text { early diastolic mitral annular velocity (E'); RVSP, right ventricular systolic pressure; LAV } \\
\text { LA volume index. }\end{array}$} \\
\hline
\end{tabular}



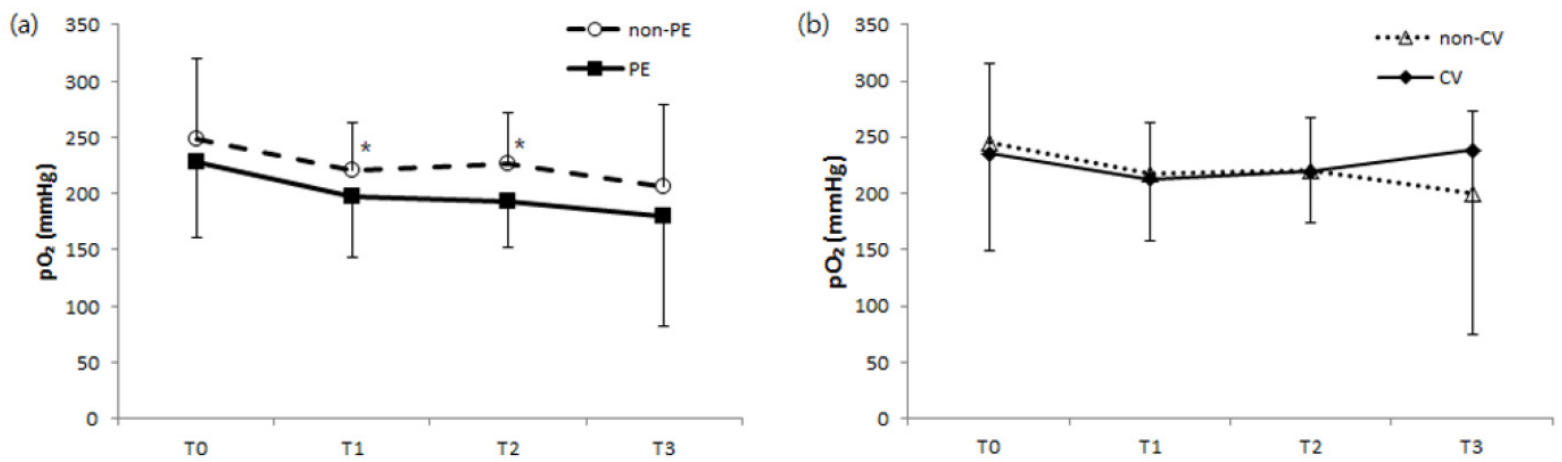

Figure 1. Comparison of changes in arterial oxygen pressure during kidney transplantation surgery regarding development of postoperative (a) pulmonary edema and no pulmonary edema or (b) major cardiovascular complications and no cardiovascular complications. PO2, arterial oxygen pressure; non-PE, no postoperative pulmonary edema; PE, postoperative pulmonary edema; non-CV, no major postoperative cardiovascular complications; $\mathrm{CV}$, major postoperative cardiovascular complications; T0, before surgery (baseline); T1, 60 minutes after surgery; T2, 10 minutes after kidney graft reperfusion; T3, end of surgery. $* \mathrm{P}<0.05$ compared to the PE group.

Table 2. Primary renal disease leading to ESRD.

\begin{tabular}{ll}
\hline Primary disease for ESRD & \\
\hline HTN & $88(36)$ \\
DM & $9(4)$ \\
GN & $51(21)$ \\
IgA nephropathy & $43(18)$ \\
MPGN & $2(1)$ \\
RPGN & $2(1)$ \\
Lupus nephritis & $2(1)$ \\
Immune-mediated GN & $2(1)$ \\
FSGS & $12(5)$ \\
PKD & $7(3)$ \\
No pre-transplantation biopsy & $76(31)$ \\
\hline Numbers are expressed as numbers of patients (percentage). \\
ESRD, end-stage renal disease; HTN, hypertension; DM, diabetes mellitus; GN, \\
glomerulonephritis; MPGN, membranoproliferative glomerulonerphritis; RPGN, rapidly \\
progressive glomerulonephritis; FSGS, focal segmental glomerulosclerosis; PKD, \\
polycystic kidney disease.
\end{tabular}

Hypertension was the predominant etiology $(36 \%)$ of ESRD, followed by glomerulonephritis $(21 \%)$, however majority of patients had no pre-transplantation biopsy for definite diagnosis (Table 2). Thirty-nine patients $(16 \%)$ developed postoperative pulmonary edema, and 9 patients $(4 \%)$ developed an in-hospital major cardiovascular complication. Specifically, these 9 patients experienced clinical pulmonary edema requiring endotracheal intubation or dialysis $(n=4)$, new-onset atrial fibrillation $(\mathrm{n}=2)$, myocardial infarction $(\mathrm{n}=2)$, or ventricular fibrillation $(n=1)$.

Intraoperative hemodynamics including MBP, CVP, and SVV; duration of surgery; intraoperative in-out fluid balances; and the number of patients receiving vasopressors during surgery were not significantly different between patients with respect to pulmonary edema or in-hospital major cardiovascular complication occurrence (data was not shown). ABG analysis also revealed no significant difference in $\mathrm{pH}$ or $\mathrm{PaCO}_{2}$ regarding pulmonary edema or cardiovascular complications, although we noted higher $\mathrm{PaO}_{2}$ levels in patients without pulmonary edema than in those with pulmonary edema 60 minutes after the start of surgery (T1) and at 10 minutes after kidney graft reperfusion (T2) (Fig 1).

After univariate analysis, we found that patients with in-hospital major cardiovascular complications had prolonged ischemia-reperfusion times during surgery, more frequent diabetes, elevated LVEDd, and greater LVMI, RVSP, LAVI, and $\mathrm{E} / \mathrm{E}^{\prime}$ ratios compared to patients without any of those complications. Multivariate analysis for these risk factors identified the $E / E^{\prime}$ ratio as a persistently strong independent predictor for in-hospital major cardiovascular complications (Table 3). The AUC of the $\mathrm{E} / \mathrm{E}^{\prime}$ ratio was 0.84 (95\% CI: 0.787-0.884), and ROC analysis showed the optimal E/E' cut-off value for predicting major cardiovascular complication was 13.0 with $77.8 \%$ sensitivity and $78.5 \%$ specificity (Fig 2).

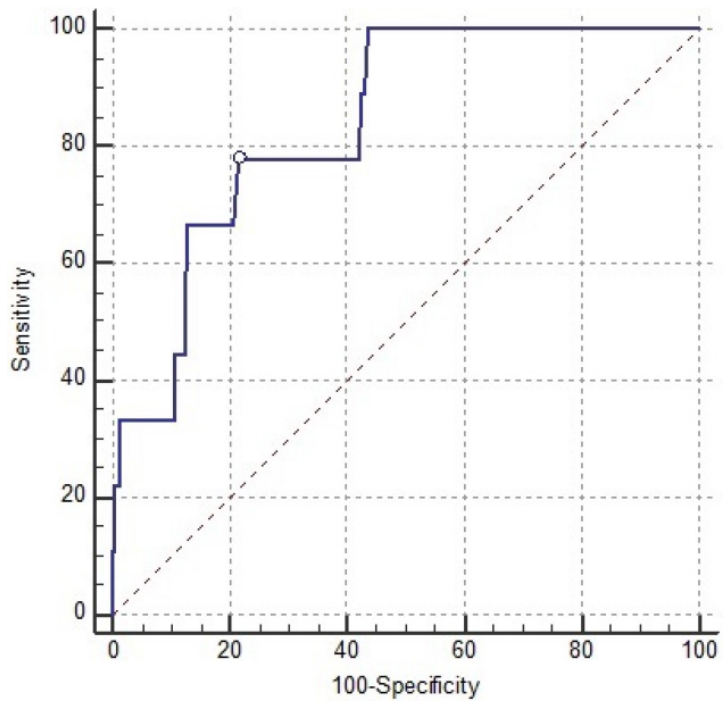

Figure 2. Receiver-operating characteristic (ROC) curve for the E/E' ratio's prediction of postoperative major cardiovascular complications. The ROC area under the ROC curve was 0.84 ( $95 \%$ confidence interval: $0.787-0.884 ; P<$ $0.001)$. 
Univariate analysis of demographic and echocardiographic data identified age, LVEDd, LVMI, LAVI, and E/E' ratios as risk factors for postoperative pulmonary edema (Table 4). However, we observed no significant differences with respect to other patient characteristics, including dialysis modalities, duration of renal replacement therapy prior to transplantation, and years diagnosed with chronic renal failure (Table 4). After subsequent multivariate analysis, no parameter remained statistically significant.

Table 3. Predictors of postoperative in-hospital major cardiovascular complications on univariate and multivariate analyses.

\begin{tabular}{|c|c|c|c|c|}
\hline & Univariate Analysis & & Multivariate Analysis & \\
\hline & OR $(95 \% \mathrm{CI})$ & $P$-value & OR $(95 \% \mathrm{CI})$ & $P$-value \\
\hline \multicolumn{5}{|l|}{ Baseline Characteristics } \\
\hline Age & $1.052(0.985-1.124)$ & 0.128 & - & \\
\hline Male & $2.098(0.549-8.022)$ & 0.279 & - & \\
\hline BMI $\left(\mathrm{kg} / \mathrm{m}^{2}\right)$ & $1.061(0.879-1.280)$ & 0.539 & - & \\
\hline \multicolumn{5}{|l|}{ Medical History } \\
\hline HTN & $1.862(0.227-15.279)$ & 0.562 & - & \\
\hline $\mathrm{DM}$ & $5.082(1.312-19.676)$ & 0.019 & $6.445(0.651-63.799)$ & 0.111 \\
\hline CAOD & $4.036(0.442-36.816)$ & 0.216 & - & \\
\hline HD vs. PD & $0.342(0.065-1.798)$ & 0.205 & - & \\
\hline Duration of CRF (yr) & $0.957(0.826-1.109)$ & 0.561 & - & \\
\hline Duration of RRT (months) & $1.006(0.993-1.019)$ & 0.382 & - & \\
\hline \multicolumn{5}{|l|}{ Operative Data } \\
\hline Op time (min) & $0.997(0.984-1.009)$ & 0.599 & - & \\
\hline I-R time (min) & $1.034(1.010-1.059)$ & 0.006 & $1.033(0.976-1.093)$ & 0.267 \\
\hline \multicolumn{5}{|l|}{ Echocardiographic data } \\
\hline $\operatorname{LVEF}(\%)$ & $0.958(0.870-1.055)$ & 0.379 & - & \\
\hline LVESd (mm) & $1.227(1.089-1.383)$ & 0.001 & $1.114(0.817-1.519)$ & 0.496 \\
\hline LVEDd (mm) & $1.282(1.112-1.477)$ & 0.001 & $1.493(0.878-2.539)$ & 0.139 \\
\hline $\operatorname{LVMI}\left(\mathrm{g} / \mathrm{m}^{2}\right)$ & $1.025(1.009-1.041)$ & 0.002 & $0.992(0.959-1.027)$ & 0.666 \\
\hline $\mathrm{E} / \mathrm{E}^{\prime}$ ratio & $1.251(1.105-1.417)$ & $<0.001$ & $1.602(1.138-2.254)$ & 0.007 \\
\hline RVSP (mmHg) & $1.064(1.007-1.124)$ & 0.027 & $0.918(0.775-1.088)$ & 0.324 \\
\hline LAVI $\left(\mathrm{mL} / \mathrm{m}^{2}\right)$ & $1.059(1.011-1.110)$ & 0.016 & $0.918(0.793-1.063)$ & 0.255 \\
\hline
\end{tabular}

Table 4. Predictors of postoperative pulmonary edema on univariate and multivariate analyses.

\begin{tabular}{|c|c|c|c|c|}
\hline & Univariate Analysis & & Multivariate Analysis & \\
\hline & OR $(95 \%$ CI $)$ & $P$-value & OR $(95 \% \mathrm{CI})$ & $P$-value \\
\hline \multicolumn{5}{|l|}{ Baseline Characteristics } \\
\hline Age & 1.037 (1.004-1.071) & 0.028 & 1.029 (0.994-1.066) & 0.106 \\
\hline Male & $1.162(0.578-2.337)$ & 0.673 & - & - \\
\hline BMI $\left(\mathrm{kg} / \mathrm{m}^{2}\right)$ & $1.102(0.997-1.218)$ & 0.056 & - & - \\
\hline \multicolumn{5}{|l|}{ Medical History } \\
\hline HTN & $2.215(0.745-6.585)$ & 0.153 & - & - \\
\hline $\mathrm{DM}$ & $1.601(0.735-3.487)$ & 0.236 & - & - \\
\hline CAOD & $1.775(0.345-9.134)$ & 0.493 & - & - \\
\hline HD vs. PD & $1.030(0.331-3.206)$ & 0.959 & - & - \\
\hline Duration of RRT (months) & $1.000(0.991-1.009)$ & 0.941 & & \\
\hline Duration of CRF (yr) & $0.953(0.884-1.028)$ & 0.215 & - & - \\
\hline \multicolumn{5}{|l|}{ Operative Data } \\
\hline Op time (min) & $1.000(0.995-1.006)$ & 0.977 & - & - \\
\hline I-R time (min) & $1.001(0.985-1.017)$ & 0.951 & - & - \\
\hline \multicolumn{5}{|l|}{ Echocardiographic data } \\
\hline $\operatorname{LVEF}(\%)$ & $0.995(0.943-1.051)$ & 0.865 & - & - \\
\hline LVESd (mm) & 1.057 (0.982-1.137) & 0.141 & - & - \\
\hline LVEDd (mm) & 1.087 (1.009-1.171) & 0.029 & $1.060(0.963-1.168)$ & 0.233 \\
\hline LVMI (g/m²) & $1.012(1.003-1.022)$ & 0.014 & 1.004 (0.990-1.019) & 0.574 \\
\hline $\mathrm{E} / \mathrm{E}^{\prime}$ ratio & $1.090(1.016-1.169)$ & 0.016 & 1.041 (0.951-1.141) & 0.385 \\
\hline RVSP (mmHg) & 1.034 (0.994-1.075) & 0.098 & - & - \\
\hline LAVI (mL/m²) & 1.029 (1.001-1.058) & 0.044 & $0.996(0.956-1.038)$ & 0.861 \\
\hline
\end{tabular}

Numbers are expressed as odds ratio (95\% Confidence Interval).

OR, odds ratio; CI confidence interval; BMI, body mass index; HD, hemodialysis; PD, peritoneal dialysis; CRF, chronic renal failure; RRT, renal replacement therapy; I-R time,

ischemia-reperfusion time, LVEF, LV ejection fraction; LVESd, LV end-systolic dimension; LVEDd, LV end-diastolic dimension; LVMI, LV mass index; E/E' ratio, the ratio of mitral peak velocity of early filling (E) to early diastolic mitral annular velocity (E'); RVSP, right ventricular systolic pressure; LAVI, LA volume index. 
Table 5. Postoperative Outcomes.

\begin{tabular}{|c|c|c|c|c|c|c|}
\hline & \multicolumn{3}{|c|}{ In-hospital major cardiovascular complications } & \multicolumn{3}{|c|}{ Pulmonary edema } \\
\hline & Yes $(n=9)$ & No $(n=233)$ & $P$-value & Yes $(n=39)$ & No $(n=203)$ & $P$-value \\
\hline DGF & $2(22)$ & $10(4)$ & 0.015 & $3(8)$ & $9(4)$ & 0.392 \\
\hline ARE & $3(33)$ & $20(9)$ & 0.013 & $5(13)$ & $18(9)$ & 0.442 \\
\hline Graft Loss & $1(11)$ & $3(1)$ & 0.024 & $2(5)$ & $2(1)$ & 0.064 \\
\hline Length of Hospital Stay (days) & $17.0 \pm 5.4$ & $15.6 \pm 5.9$ & 0.477 & $17.5 \pm 10.2$ & $15.3 \pm 4.5$ & 0.033 \\
\hline
\end{tabular}

Numbers are expressed as numbers of patients (percentage), or means \pm SD.

DGF, delayed graft function; ARE, acute rejection episode.
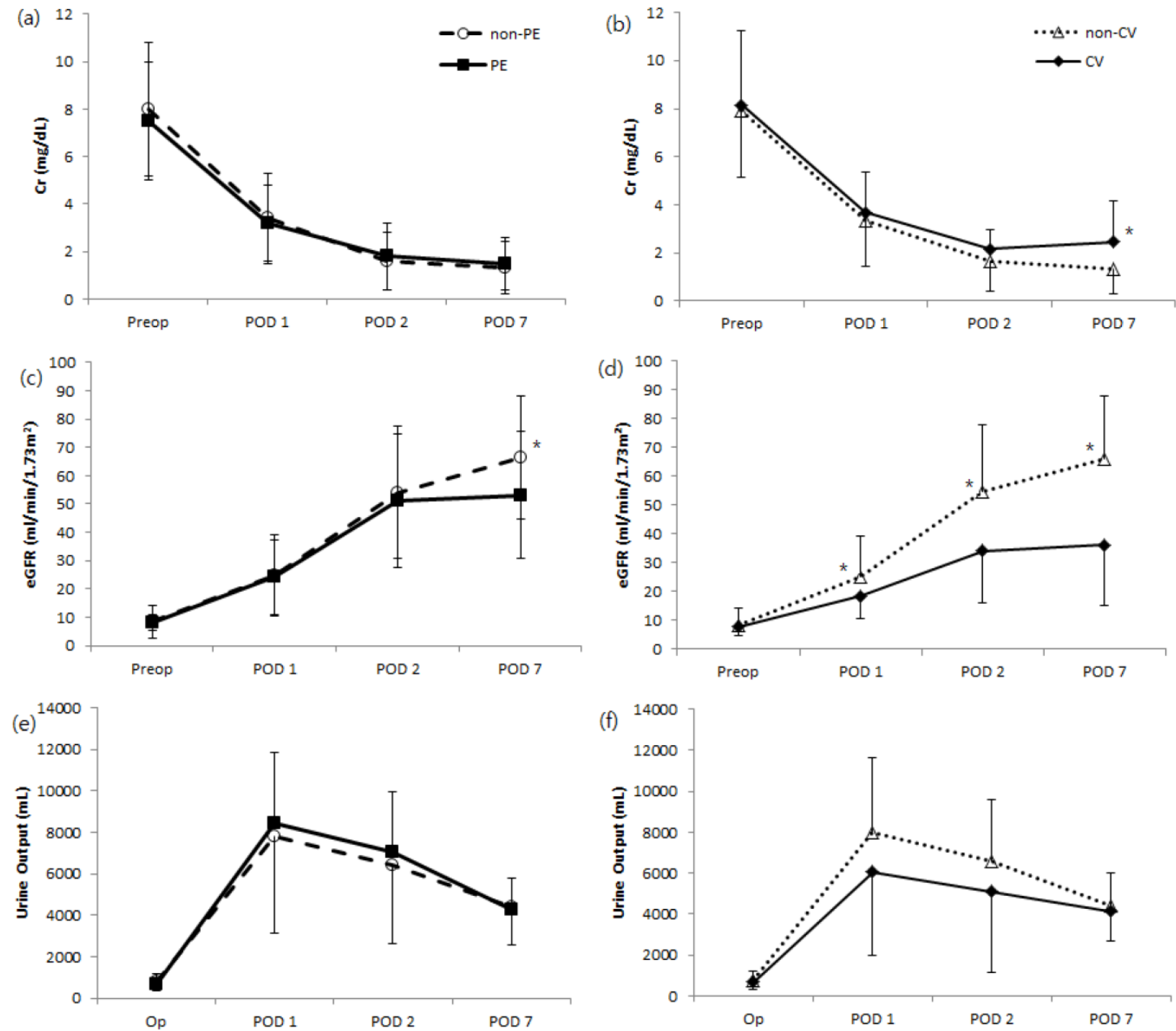

Figure 3. Comparison of changes in postoperative renal function as indicated by means of creatinine (Cr), estimated glomerular filtration rate (eGFR), and daily urine output after kidney transplantation. Their relationship to the development of postoperative (a, c, e) pulmonary edema and no pulmonary edema or (b, $d$, f) major cardiovascular complications and no cardiovascular complications are shown. non-PE, no postoperative pulmonary edema; PE, postoperative pulmonary edema; non-CV, no major postoperative cardiovascular complications; $\mathrm{CV}$, major postoperative cardiovascular complications. $* P<0.05$ compared to either the PE or $\mathrm{CV}$ group.

Postoperative renal function significantly increased, as indicated by serum $\mathrm{Cr}$ on postoperative day 7 , in the major cardiovascular complication group compared to patients without complications. Levels of eGFR increased significantly during postoperative day 7 in patients without major cardiovascular complications or pulmonary edema, while the amount of daily urine output was not significantly different with respect to postoperative cardiovascular complications or pulmonary outcome (Fig 3). Greater percentages of patients with major cardiovascular complications were associated with DGF, ARF or graft loss altogether, compared to patients without such complications (Table 5). We also noted a significant difference in mean length of hospital stay according to the development of pulmonary edema $(P$ $=0.033$ ) but not occurrence of in-hospital major cardiovascular complications (Table 5). One 
in-hospital mortality occurred after transplantation during the study period, in which the patient died from multi-organ failure on postoperative day 74 .

\section{Discussions}

We evaluated the utility of echocardiographic parameters for predicting postoperative cardiopulmonary events in patients undergoing living donor kidney transplantation. We found that a greater preoperative $\mathrm{E} / \mathrm{E}^{\prime}$ ratio, a reliable indicator of LV diastolic dysfunction, was significantly related to the development of major cardiovascular complications in kidney recipients during a defined postoperative period.

Cardiac structure and function alterations in patients with chronic kidney disease have been extensively studied, leading to a growing appreciation of the impact of cardiovascular abnormalities on morbidity and mortality in ESRD patients $[1,5,27]$. The pathophysiological characteristics of these abnormalities in chronic kidney disease and ESRD involve hemodynamic overload from arteriovenous shunts, arterial remodeling, and anemia, as well as metabolic changes, such as uremic toxicity, renin-angiotensin-aldosterone system hyperactivity, and secondary hyperparathyroidism [2-4]. Through these diverse mechanisms, even during early progressive chronic kidney disease, myocardial hypertrophy and fibrosis lead to alterations in LV relaxation and compliance and ultimately to the development of LV diastolic dysfunction [13]. The prevalence of this dysfunction evaluated by echocardiography in ESRD patients ranges from $30-75 \%$, depending on the criteria used for its quantification $[6,9,13]$. Furthermore, LV hypertrophy and shifted LV pressure-volume curves exacerbate the effects of both blood volume changes on LV filling pressure and arrhythmia on hemodynamic instability [28]. The prognostic impact of diastolic dysfunction on clinical morbidities, such as pulmonary edema, major cardiovascular complications, or even death, has been demonstrated in various populations of patients $[12,16]$. Fifty percent of ESRD patients in their first year of hemodialysis experienced mild diastolic dysfunction, and $23 \%$ of patients presented with pseudo normalization or restrictive flow pattern predictive of cardiovascular events (hazard ratio 2.2), regardless of age, gender, diabetes, LV mass, or ejection fraction [13]. However, limited information exists regarding the relationship between diastolic dysfunction and cardiopulmonary complications in ESRD patients undergoing kidney transplantation. Thus, we evaluated the impact of preoperative diastolic dysfunction on the occurrence of major cardiovascular complications and postoperative pulmonary edema in ESRD patients after living donor kidney transplantation using tissue Doppler imaging.

Among various relevant echocardiographic parameters, the role of tissue Doppler echocardiography in predicting diastolic dysfunction has been explored previously $[29,30]$. Specifically, the $\mathrm{E} / \mathrm{E}^{\prime}$ ratio is a relatively independent preload parameter that correlates with LV filling pressure [19] and predicts certain cardiovascular outcomes, such as cardiomyopathy, acute myocardial infarction, and atrial fibrillation $[14,15,29]$. For example, an E/E' ratio of $<8$ or $>15$ accurately predicts normal or increased mean LV diastolic pressure, respectively, whereas an $\mathrm{E} / \mathrm{E}^{\prime}$ ratio between 8 and 15 shows poor correlation [17,31,32]. Additionally, an $\mathrm{E} / \mathrm{E}^{\prime}$ ratio greater than 15 reliably predicts mortality $[19,33]$. In previous studies evaluating the cardiovascular effects of successful kidney transplantation, LV hypertrophy and systolic dysfunction resolved after transplantation, but data regarding the impact of transplant toward diastolic dysfunction are controversial [5-7]. Interestingly, analyses limited to use of transmitral flow-derived Doppler parameters identified progressive LV diastolic dysfunction, despite improvement of systolic function and LV hypertrophy after successful transplantation [6,8]. In contrast, studies assessing diastolic function in terms of $\mathrm{E} / \mathrm{E}^{\prime}$ ratio have shown improved diastolic function in concordance with alterations in systolic function and LV mass [5,7]. In this context, our current study determined that prolonged ischemia-reperfusion time, diagnosis of diabetes, elevated LVEDd, and greater LVMI, RVSP, LAVI, and $\mathrm{E} / \mathrm{E}^{\prime}$ ratio were significant risk factors for in-hospital major cardiovascular complications, with $\mathrm{E} / \mathrm{E}^{\prime}$ ratio strongly correlating with the development of adverse cardiovascular complications after multivariate analysis. Moreover, ROC analysis identified 13.0 as the optimal cut-off value of the $E / E^{\prime}$ ratio for predicting major cardiovascular complications with an accompanying AUC of 0.84, which corroborates previous reports that found an $\mathrm{E} / \mathrm{E}^{\prime}$ ratio greater than 15 closely relates to patient morbidities.

Achieving optimal fluid management therapy for ESRD patients undergoing kidney transplantation is critical for maintaining adequate intravascular volume to enhance graft function and avoid fluid overload [34,35], especially because the transplanted kidney is denervated and lacks autoregulation [36]. Deleterious effects of fluid overload on cardiovascular and pulmonary physiology include impaired cardiac output and related morbidities, so various attempts to establish a standard management strategy during and after kidney transplantation have been made. The 
most commonly adopted management principle is CVP due to its ability to indirectly reflect a patient's volume status, although goal-direct fluid therapy targets SVV to guide fluid management and may be superior to traditional CVP monitoring [37]. However, we found that patients showed changes in MBP, HR, CVP, and SVV during the perioperative period, regardless of postoperative pulmonary edema and major cardiovascular complication occurrence, highlighting the limitations of hemodynamic parameters to predict and prevent the development of post-transplant cardiopulmonary complications. Echocardiography, a highly precise tool for evaluating volume status during various types of surgery, is a more reliable predictor of such complications compared to the hemodynamic parameters mentioned above. As the importance of echocardiography in fluid management continues to be emphasized, more comprehensive preoperative cardiac work-ups for every transplant candidate should be performed to provide an individualized strategy for proper goal-directed therapy.

We also identified age, LVEDd, LVMI, LAVI, and $E / E^{\prime}$ ratio as risk factors for postoperative pulmonary edema. Unexpectedly, pulmonary edema diagnosed with postoperative chest $x$-rays only weakly correlated with preoperative echocardiographic parameters and postoperative prognosis, such as DGF, ARE, and graft loss. In contrast, in-hospital major cardiovascular complications, including clinical pulmonary edema requiring endotracheal intubation or dialysis, strongly correlated with certain diastolic dysfunction-related echocardiographic parameters and postoperative deterioration of graft function. Such correlations can be inferred from the inevitable causal relationship between overloaded volume status of ESRD patients and their diastolic dysfunction, which can worsen volume overload and result in unfavorable cardiorespiratory and graft outcomes. Study patients who developed perioperative pulmonary edema also exhibited characteristic ABG findings consistent with pulmonary edema, such as low $\mathrm{PaO}_{2}$, even when baseline oxygenation levels were not significantly different.

One limitation of the current study is its observational nature, which may promote study bias. This study only included patients selected for living donor kidney transplantation with well-qualified 2-months preoperative echocardiographic data during the study period, which might have influenced the prognostic conclusions that could be drawn from our analysis. Moreover, we could not control for the timing of preoperative echocardiograms, so possible variations in intravascular volume may have affected the echocardiographic data of ESRD patients on hemodialysis. In addition, our study population may not be consistent demographically and/or clinically with patients from previous studies with respect to progression of LV systolic dysfunction. In this study, only one patient experienced moderate LV systolic dysfunction, and none presented with severe LV systolic dysfunction per preoperative echocardiography. Thus, our emphasis on diastolic, rather than systolic, dysfunction may be contrary to findings from previous studies, which focused on the prognostic value of systolic dysfunction after kidney transplantation [5,38]. The patients enrolled in our study were relatively younger compared to those of previous studies, as most of our patients were scheduled for preemptive kidney transplantation before full-blown kidney failure. Such different biased distribution of patient age may have been the reason for the unique patient presentation in the present study, which could have affected the absence of age-related contributions on postoperative complications. Lastly, we followed patient prognosis during the initial post-transplant hospital stay only, which can be relatively short, while other studies incorporated long-term evaluation periods for graft outcomes and patient prognosis.

In conclusion, subclinical LV diastolic dysfunction as indicated by a high $\mathrm{E} / \mathrm{E}^{\prime}$ ratio can consistently predict the occurrence of in-hospital major cardiovascular complications in living donor kidney recipients. Based on our results, we propose that ESRD patients with preexisting subclinical diastolic dysfunction who will undergo living donor kidney transplantation be carefully monitored for volume and hemodynamic imbalances during the perioperative period.

\section{Abbreviations}

ESRD: end-stage renal disease; LVEDd: left ventricular end-diastolic diameter; LVMI: left ventricular mass index; RVSP: right ventricular systolic pressure; LAVI: left atrium volume index; LV: left ventricle; E/E': ratio of early transmitral flow velocity to early diastolic velocity of the mitral annulus; MBP: mean blood pressure; HR: heart rate; CVP: central venous pressure; SVV: stroke volume variation; $A B G$ : arterial blood gas; E/A: ratio of peak early and late (atrial) mitral inflow; DGF: delayed graft function; ARE: acute rejection episodes; $\mathrm{Cr}$ : creatinine; eGFR: estimated glomerular filtration rate; SD: standard deviation; ROC: receiver-operating characteristic; AUC: area under the curve; OR: odds ratio; CI: confidence interval; BMI: body mass index; HD: hemodialysis; PD: peritoneal dialysis; CRF: 
chronic renal failure; RRT: renal replacement therapy; $\mathrm{Hb}$ : hemoglobin; I-R time: ischemia-reperfusion time.

\section{Acknowledgements}

This research was supported by Basic Science Research Program through the National Research Foundation of Korea (NRF) funded by the Ministry of Science, ICT \& Future Planning (NRF2014R1A1A3053428)

\section{Competing Interests}

The authors have declared that no competing interest exists.

\section{References}

1. Parfrey PS, Foley RN. The clinical epidemiology of cardiac disease in chronic renal failure. J Am Soc Nephrol. 1999; 10(7): 1606-15.

2. Foley RN, Parfrey PS, Harnett JD, Kent GM, Murray DC, Barré PE. The prognostic importance of left ventricular geometry in uremic cardiomyopathy. J Am Soc Nephrol. 1995; 5(12): 2024-31.

3. Wolfe RA, Ashby VB, Milford EL, Ojo AO, Ettenger RE, Agodoa LY, et al. Comparison of mortality in all patients on dialysis, patients on dialysis awaiting transplantation, and recipients of a first cadaveric transplant. N Engl J Med. 1999; 341(23): 1725-30.

4. Parfrey PS, Foley RN, Harnett JD, Kent GM, Murray D, Barre PE. Outcome and risk factors of ischemic heart disease in chronic uremia. Kidney Int. 1996; 49(5): 1428-34.

5. Hawwa N, Shrestha K, Hammadah M, Yeo PS, Fatica R, Tang WH. Reverse Remodeling and Prognosis Following Kidney Transplantation in Contemporary Patients With Cardiac Dysfunction. J Am Coll Cardiol. 2015; 66(16): 1779-87.

6. Ferreira SR, Moisés VA, Tavares A, Pacheco Silva A. Cardiovascular effects of successful renal transplantation: a 1-year sequential study of left ventricular morphology and function, and 24-hour blood pressure profile. Transplantation. 2002; 74(11): 1580-7.

7. de Souza FL, Bezerra KB, Sousa AR, Ferreira TC, Oliveira MI, Martins GP, et al. Study of echocardiographic alterations in the first six months after kidney transplantation. Arq Bras Cardiol. 2012; 98(6): 505-13

8. Dudziak M, Debska Slizień A, Rutkowski B. Cardiovascular effects of successful renal transplantation: a 30-month study on left ventricular morphology, systolic and diastolic functions. Transplant Proc. 2005; 37(2): 1039-43.

9. Stewart GA, Gansevoort RT, Mark PB, Rooney E, McDonagh TA, Dargie HJ, et al. Electrocardiographic abnormalities and uremic cardiomyopathy. Kidney Int. 2005; 67(1): 217-26.

10. Glassock RJ P-FR, Barberato SH. Left ventricular mass in chronic kidney disease and ESRD. Clin J Am Soc Nephrol. 2009; 4(Suppl 1): S79-91.

11. GM L. Left ventricular alterations and end-stage renal disease. Nephrol Dial Transplant. 2002; 17(Suppl 1): 29-36.

12. Pecoits Filho R, Bucharles S, Barberato SH. Diastolic heart failure in dialysis patients: mechanisms, diagnostic approach, and treatment. Semin Dial. 2012; 25(1): 35-41.

13. Barberato SH, Bucharles SG, Sousa AM, Costantini CO, Costantini CR, Pecoits Filho R. [Prevalence and prognostic impact of diastolic dysfunction in patients with chronic kidney disease on hemodialysis]. Arq Bras Cardiol. 2010; 94(4): 457-62.

14. Lee E, Yun S, Chin J, Choi D, Son H, Kim W, et al. Prognostic implications of preoperative $\mathrm{E} / \mathrm{e}^{\prime}$ ratio in patients with off-pump coronary artery surgery. Anesthesiology. 2012; 116(2): 362-71.

15. Cho D, Park S, Kim M, Kim SA, Lim H, Shim W. Presence of preoperative diastolic dysfunction predicts postoperative pulmonary edema and cardiovascular complications in patients undergoing noncardiac surgery. Echocardiography. 2014; 31(1): 42-9.

16. Higashi M, Yamaura K, Ikeda M, Shimauchi T, Saiki H, Hoka S. Diastolic dysfunction of the left ventricle is associated with pulmonary edema after renal transplantation. Acta Anaesthesiol Scand. 2013; 57(9): 1154-60.

17. Nagueh SF, Appleton CP, Gillebert TC, Marino PN, Oh JK, Smiseth OA, et al. Recommendations for the evaluation of left ventricular diastolic function by echocardiography. J Am Soc Echocardiogr. 2009; 22(2): 107-33.

18. Wang AY, Wang M, Lam CW, Chan IH, Zhang Y, Sanderson JE. Left ventricular filling pressure by Doppler echocardiography in patients with end-stage renal disease. Hypertension. 2008; 52(1): 107-14

19. Sharma R, Pellerin D, Gaze DC, Mehta RL, Gregson H, Streather CP, et al. Mitral peak Doppler E-wave to peak mitral annulus velocity ratio is an accurate estimate of left ventricular filling pressure and predicts mortality in end-stage renal disease. J Am Soc Echocardiogr. 2006; 19(3): 266-73.
20. Friedewald J, Reese P. The kidney-first initiative: what is the current status of preemptive transplantation? Adv Chronic Kidney Dis. 2012; 19(4): 252-6.

21. Nishimura RA, Otto CM, Bonow RO, Carabello BA, Erwin JP, Guyton RA, et al. 2014 AHA/ACC guideline for the management of patients with valvular heart disease: a report of the American College of Cardiology/ American Heart Association Task Force on Practice Guidelines. J Am Coll Cardiol. 2014; 63(22): e57-185.

22. Lang RM, Badano LP, Mor Avi V, Afilalo J, Armstrong A, Ernande L, et al. Recommendations for cardiac chamber quantification by echocardiography in adults: an update from the American Society of Echocardiography and the European Association of Cardiovascular Imaging. J Am Soc Echocardiogr. 2015; 28(1): 1-39.e14

23. Khouri SJ, Maly GT, Suh D, Walsh TE. A practical approach to the echocardiographic evaluation of diastolic function. J Am Soc Echocardiogr. 2004; 17(3): 290-7.

24. Taber DJ, McGillicuddy JW, Bratton CF, Lin A, Chavin KD, Baliga PK. The concept of a composite perioperative quality index in kidney transplantation. J Am Coll Surg. 2014; 218(4): 588-97.

25. Thygesen K, Alpert JS, Jaffe AS, Simoons ML, Chaitman BR, White HD, et al. Third universal definition of myocardial infarction. Circulation. 2012; 126(16): 2020-35

26. Lee JH, Joo DJ, Kim JM, Park JH, Kim YS, Koo BN. Preconditioning effects of the anesthetic administered to the donor on grafted kidney function in living donor kidney transplantation recipients. Minerva Anestesiol. 2013; 79(5): 504-14.

27. Meeus F KO, Guerin AP, Gaudry C, Marchais SJ, London GM. Pathophysiology of cardiovascular disease in hemodialysis patients. Kidney Int. 2000; 76: S140-7.

28. Kitzman DW, Little WC, Brubaker PH, Anderson RT, Hundley WG, Marburger CT, et al. Pathophysiological characterization of isolated diastolic heart failure in comparison to systolic heart failure. JAMA. 2002; 288(17): 2144-50.

29. Saito S, Takagi A, Kurokawa F, Ashihara K, Hagiwara N. Usefulness of tissue Doppler echocardiography to predict perioperative cardiac events in patients undergoing noncardiac surgery. Heart Vessels. 2012; 27(6): 594-602.

30. Shim JK, Choi YS, Chun DH, Hong SW, Kim DH, Kwak YL. Relationship between echocardiographic index of ventricular filling pressure and intraoperative haemodynamic changes during off-pump coronary bypass surgery. Br J Anaesth. 2009; 102(3): 316-21.

31. Ommen SR, Nishimura RA, Appleton CP, Miller FA, Oh JK, Redfield MM, et al. Clinical utility of Doppler echocardiography and tissue Doppler imaging in the estimation of left ventricular filling pressures: A comparative simultaneous Doppler-catheterization study. Circulation. 2000; 102(15): 1788-94.

32. Paulus WI, Tschöpe $C$, Sanderson JE, Rusconi $C$, Flachskampf FA, Rademakers FE, et al. How to diagnose diastolic heart failure: a consensus statement on the diagnosis of heart failure with normal left ventricular ejection fraction by the Heart Failure and Echocardiography Associations of the European Society of Cardiology. Eur Heart J. 2007; 28(20): 2539-50.

33. Chen S, Chang J, Tsai Y, Huang J, Chen LI, Su H, et al. Ratio of transmitral E-wave velocity to early diastole mitral annulus velocity with cardiovascular and renal outcomes in chronic kidney disease. Nephron Clin Pract. 2013; 123(1-2): 52-60

34. Schnuelle P, Johannes van der Woude F. Perioperative fluid management in renal transplantation: a narrative review of the literature. Transpl Int. 2006; 19(12): 947-59.

35. De Gasperi A, Narcisi S, Mazza E, Bettinelli L, Pavani M, Perrone L, et al. Perioperative fluid management in kidney transplantation: is volume overload still mandatory for graft function? Transplant Proc. 2006; 38(3): 807-9.

36. Campos L, Parada B, Furriel F, Castelo D, Moreira P, Mota A. Do intraoperative hemodynamic factors of the recipient influence renal graft function? Transplant Proc. 2012; 44(6): 1800-3.

37. Chin JH, Jun IG, Lee J, Seo H, Hwang GS, Kim YK. Can stroke volume variation be an alternative to central venous pressure in patients undergoing kidney transplantation? Transplant Proc. 2014; 46(10): 3363-6.

38. Wali RK, Wang GS, Gottlieb S, Bellumkonda L, Hansalia R, Ramos E, et al. Effect of kidney transplantation on left ventricular systolic dysfunction and congestive heart failure in patients with end-stage renal disease. J Am Coll Cardiol. 2005; 45(7): 1051-60. 